\title{
Seasonal microbial community dynamics in a flowerpot-using personal composting system for disposal of household biowaste
}

\author{
Akira Hiraishi, ${ }^{*}$ Yosuke Yamanaka, and Takashi Narihiro \\ Department of Ecological Engineering, Toyohashi University of Technology, Toyohashi 441-8580, Japan
}

(Received May 8, 2000; Accepted August 11, 2000)

\begin{abstract}
Microbial community dynamics in a flowerpot-using solid biowaste composting (FUSBIC) process were monitored seasonally by quinone profiling and conventional microbiological methods. The FUSBIC system, which consisted of three flowerpots ( $14 \mathrm{~L}$ or $20 \mathrm{~L}$ capacity) with 5-6 kg each of a soil-compost mixture (SCM) as the primary reactors, was loaded daily with household biowaste from November 1998 to October 1999. The monthly average waste reduction rate was $88.2 \%$ for the $14-\mathrm{L}$ system and $92.5 \%$ for the 20 - L system on a wet weight basis. The direct total microbial count detected in the 14-L primary reactors ranged from 4.5 to $9.6 \times 10^{11}$ cells $\cdot \mathrm{g}^{-1}$ of dry wt of SCM, and the viable count of aerobic heterotrophic bacteria recovered on agar plates at $28^{\circ} \mathrm{C}$ varied from 1.9 to $5.7 \times 10^{11} \mathrm{CFU} \cdot \mathrm{g}^{-1}$ of dry wt. The quinone content of SCM samples from the $14-\mathrm{L}$ and $20-\mathrm{L}$ systems ranged from 160 to $353 \mathrm{nmol}^{-\mathrm{g}^{-1}}$ of dry SCM. Ubiquinones, unsaturated menaquinones, and partially saturated menaquinones constituted 15.0-36.4, 14.8-22.0, and 41.8-61.6 mol\% of the total content, respectively. The major quinone types detected were usually MK-8 $\left(\mathrm{H}_{2}\right)$, MK-9( $\left.\mathrm{H}_{2}\right)$, and Q-10. Variations in quinone profiles were evaluated numerically by using two parameters, the dissimilarity index $(D)$ and microbial divergence index $\left(M D_{\mathrm{q}}\right)$. The upper limit of seasonal changes in the microbial community structure was about $30 \%$ as expressed by $D$ values. The $M D_{\mathrm{q}}$ values calculated ranged from 18 to 22. A significant positive correlation was found between seasonal temperature and bacterial populations containing partially saturated menaquinones. These results indicated that the FUSBIC system contained highly diverse microbial populations that fluctuated to some extent depending on seasonal temperature. Members of the Actinobacteria were suggested to be the major constituents of the total population present.
\end{abstract}

Key Words_biowaste; compost; microbial community; quinone profiles

Introduction

Composting is a subject of major concern in municipal waste management involved with the disposal of household organic waste. In Japan, personal or smallscale composting processes for household biowaste management have received much attention, and sev-

${ }^{*}$ Address reprint requests to: Dr. Akira Hiraishi, Department of Ecological Engineering, Toyohashi University of Technology, Tenpaku-cho, Toyohashi 441-8580, Japan.

E-mail: hiraishi@eco.tut.ac.jp eral electric and nonelectric composters for personal use are now commercially available. In the conventional composting system in a long-term batch process, solid organic waste is degraded to soluble low molecules and turned into stable organic compounds containing a humic-like substance by passing through four major thermal stages, i.e., the mesophilic, thermophilic, cooling, and maturation stages. At each thermal stage, specific microorganisms predominate and play a primary role in the reduction and conversion of organic waste in response to temperature (for reviews, Finstein and Morris, 1975; Fogarty and Tuovi- 
nen, 1991). On the other hand, the personal composting system using an electric or nonelectric composter is a continuous daily batch process without definitive thermal stages. Therefore this system may differ from the traditional composting process in microbial community dynamics. Although large numbers of microbiological studies have been performed on various composting systems, the available information on the microbiology of continuous batch processes for composting household biowaste is limited and fragmentary.

Previously (Hiraishi, 1999a, 2000), we reported the development of a flowerpot-using solid biowaste composting (FUSBIC) process and its application to the composting of household biowaste. The primary reactors of this process are capable of reducing added biowaste to less than $10 \%$ on a wet weight basis when operated in the sun at a waste loading rate of less than about $0.2 \mathrm{~kg}$ (wet wt) $\cdot$ reactor ${ }^{-1} \cdot \mathrm{d}^{-1}$. A preliminary microbiological study has shown that microorganisms at the order of $10^{11}$ cells $\cdot \mathrm{g}^{-1}$ of dry soil are present in the reactors, and members of the Actinobacteria may be the major constituents of the microbial population. A more comprehensive understanding of microbial community dynamics is needed not only to improve the FUSBIC process, but also to provide basic information of value in the development and engineering of other composting systems for personal use.

In recent years, culture-independent approaches have been conducted for the assessment of microbial diversity and community structure in compost. These approaches include the characterization of microbial communities based on PCR-aided molecular analyses (Kowalchuk et al., 1999; Peters et al., 2000), carbon source utilization patterns (Carpenter-Boggs et al., 1998; Insam et al., 1996), and phospholipid fatty acid profiles (Carpenter-Boggs et al., 1998; Hellmann et al., 1997; Herrmann and Shann, 1997; Klamer and Bååth, 1998). Respiratory quinone profiling is among the most useful culture-independent biomarker approaches for studying complex microbial communities in compost because of its applicability to the simultaneous analysis of prokaryotic and eukaryotic microorganisms and its technical advantages, i.e., simplicity, high reproducibility, and ease in data interpretation (Hiraishi, 1999b). This biomarker approach has been successfully applied to characterize microbial communities in compost and soil environments (Fujie et al., 1998; Hiraishi, 1999a, 2000; Iwasaki and Hiraishi, 1998; Katayama et al., 1998). In this study, we used the quinone profiling technique and conventional microbiological methods for monitoring seasonal microbial community dynamics in the FUSBIC process. A numerical analysis of profiles was made to obtain quantitative information on population shifts over time.

\section{Materials and Methods}

Composting system. The FUSBIC system usually used for a family of 3 or 4 people consists of three polypropylene flowerpots ( $14 \mathrm{~L}$ or $20 \mathrm{~L}$ capacity) as primary reactors and one or two as secondary reactors (Hiraishi, 1999a). In this study, two FUSBIC systems with commercially available 14-L and 20-L flowerpots (Richell Co., Toyama, Japan) were used in parallel. Each flowerpot for the primary treatment was packed successively with $2.0 \mathrm{~kg}$ of fine gravel, $1.0 \mathrm{~kg}$ of beaded red clay, and 5.0 to $6.0 \mathrm{~kg}$ of a soil-compost mixture (SCM) (Fig. 1a). The SCM was prepared by mixing an equal weight of garden soil (Compost Kogyo, Gifu, Japan) and the mature compost that was produced previously in the FUSBIC process (Hiraishi, 1999a). Thus the total weight of each reactor was

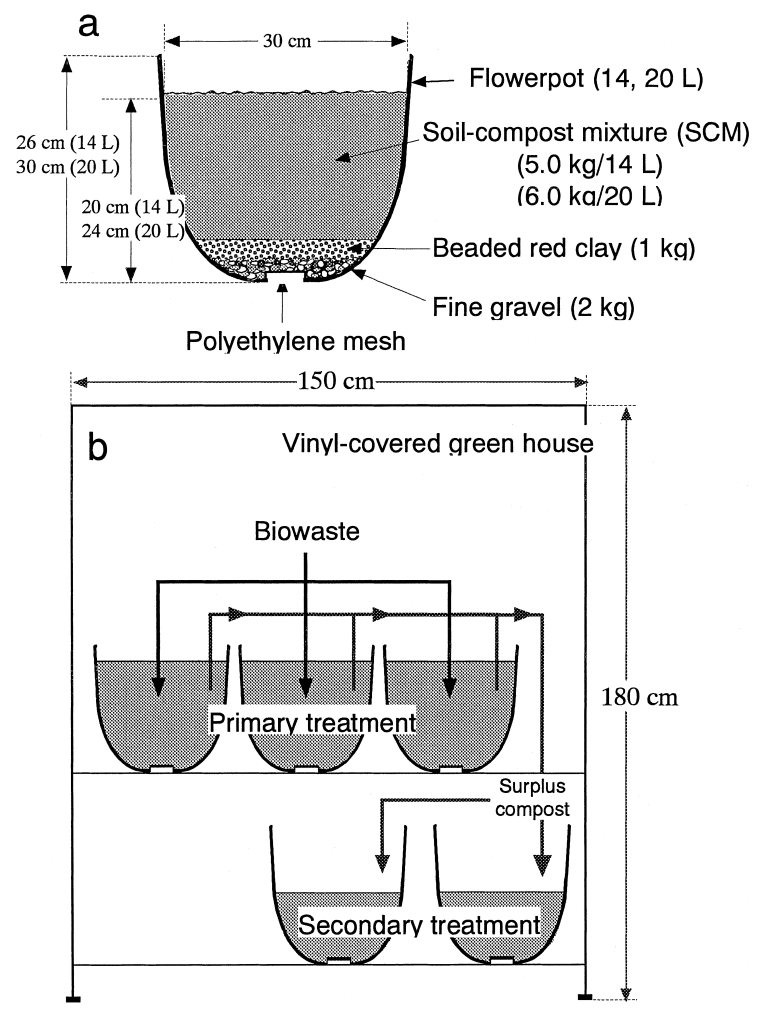

Fig. 1. Schematic illustration of the FUSBIC system.

(a) Cross-section of a primary reactor, (b) reactors settled in a vinyl-covered greenhouse. 
Table 1. Composition of household biowaste used for a FUSBIC system during one year.

\begin{tabular}{lcr}
\hline \multicolumn{1}{c}{ Source and content } & Wet weight $(\mathrm{kg})$ & $\%$ \\
\hline Dark-green vegetables & 35.1 & 14.6 \\
Yellowish-green vegetables & 48.1 & 20.0 \\
Citrus and fruits & 41.7 & 17.3 \\
Root crops & 19.2 & 8.0 \\
Tea/coffee grounds & 25.7 & 10.7 \\
Leftover rice/bread crumbs & 19.0 & 7.9 \\
Fish/shells & 13.6 & 5.6 \\
Meat & 2.2 & 0.9 \\
Eggshells & 10.4 & 4.3 \\
Others & 26.0 & 10.8
\end{tabular}

$8.7 \mathrm{~kg}$ for $14-\mathrm{L}$ flowerpots and $9.9 \mathrm{~kg}$ for $20-\mathrm{L}$ ones. All reactors were settled in a vinyl-covered personal greenhouse to shut out the rain (Fig. 1b), but the vinyl cover was opened on fine days, as reported previously (Hiraishi, 2000).

Biowaste. Organic waste disposed from the author's (A.H.) family was collected over a year and used for composting. The biowaste from the restaurant of Toyohashi University of Technology was also sometimes used. The average composition of the organic waste used is shown in Table 1. Before use, biowaste samples were cut into pieces ( $\leq 3 \mathrm{~cm}$ square), and water of the waste was squeezed as possible. The average moisture content of the waste thus obtained was $77.8 \%$. The average $\mathrm{pH}$ was 6.07 .

Operation of reactors. The primary reactors of the 14-L and 20-L systems were loaded daily with biowaste of an average wet weight of $0.655 \mathrm{~kg} \cdot \mathrm{d}^{-1}$, in which the waste was added twice to the reactors, at 8 a.m. and 9 p.m. The loading rate noted above corresponded to $0.218 \mathrm{~kg} \cdot$ reactor $^{-1} \cdot \mathrm{d}^{-1}$, and $0.043 \mathrm{~kg} \cdot \mathrm{kg}$ $\mathrm{SCM}^{-1} \cdot \mathrm{d}^{-1}$ for $14-\mathrm{L}$ reactors and $0.035 \mathrm{~kg} \cdot \mathrm{kg} \mathrm{SCM}^{-1}$. $\mathrm{d}^{-1}$ for 20-L reactors. Every time the waste was added to the reactors, it was stirred well into SCM with a trowel for about $1 \mathrm{~min}$. The reactors were operated always at ambient temperature in the sun. Surplus compost produced was removed monthly from the primary reactors by giving the original weight of the reactor and was transferred to a secondary reactor. The operation period was from November 1998 to October 1999.

Sample collection. SCM samples were collected equally from the three primary reactors, which were stirred well with a trowel just before sampling, and mixed completely. The mixture was then passed through a stainless sieve with a 3-mm mesh and taken into sterile polypropylene tubes. If pieces of biowaste and small visible organisms such as ticks and nematodes were found in samples, they were removed by forceps when possible. The samples were immediately used for microbiological analyses, whereas those for quinone profiling were stored at $-80^{\circ} \mathrm{C}$ until analyzed.

Physico-chemical analyses. The waste reduction rate was calculated by measuring the increase in weight of the reactors compared with the cumulative amount (wet wt) of biowaste added. The weights of the reactors were measured directly on a Yamato model SD-20 spring balance (Yamato Seiko Co., Tokyo, Japan) as described previously (Hiraishi, 1999). SCM temperature was measured with a digital thermometer at $8 \mathrm{~cm}$ depth in the core of the reactor as previously described (Hiraishi, 1999). The pH of SCM was measured for its suspension in distilled water.

Direct counting. Total microbial counts (TCs) were measured directly by epifluorescence microscopy with ethidium bromide staining according to the protocols of Roser (1980) and Someya (1995), with some modifications. One gram (wet wt) of SCM was suspended in $9 \mathrm{ml}$ of filter-sterilized pure water, sonicated on ice for $1 \mathrm{~min}$ with $2 \mathrm{~s}$ intermittent bursts $(20 \mathrm{kHz}$; output power, $50 \mathrm{~W}$ ), and settled for $10 \mathrm{~min}$. One milliliter of the upper fraction of this suspension was diluted decimally with the filter-sterilized pure water. An aliquot $(10-50 \mu \mathrm{l})$ of an appropriate dilution was taken into microtubes, stained with ethidium bromide in buffered solution ( $\mathrm{pH} 7.2$ ), and filtered through a $0.2 \mu \mathrm{m}$-pore Nucleopore ${ }^{\circledR}$ filter (Corning, New York, NY, USA). Ethidium bromide stained cells were detected under an Olympus BX-50 epifluorescence microscope equipped with a Flovel FD-120M digital camera (Flovel Co., Tokyo, Japan). The total count was calculated by averaging the number of cells in a random 10 fields.

Plate counting. Viable bacterial counts (VBCs) and viable mold/yeast counts (VMCs) were obtained by the plate-counting methods. One gram (wet wt) of SCM was suspended in autoclaved $1 \%$ peptone water with Bacto ${ }^{\circledR}$ Peptone (Difco Laboratories, Detroit, MI, USA), sonicated for $1 \mathrm{~min}$ as noted above, and settled for $10 \mathrm{~min}$ at room temperature. One milliliter of the upper fraction of this suspension was taken and diluted decimally with $1 \%$ peptone water. An aliquot $(20-50 \mu \mathrm{l})$ of each dilution was spread onto $1.8 \%$ agar plates containing PBYG medium (0.5\% Proteose Peptone No. 3 
[Difco], 0.1\% Beef Extract [Difco], 0.1\% Yeast Extract [Difco], $0.1 \%$ glucose, and $0.1 \% \mathrm{NaCl}, \mathrm{pH} 7.4$ ). Inoculated plates were incubated in air incubators at 28, 37, and $50^{\circ} \mathrm{C}$ for 7 days before counting colony-forming units (CFU) as VBCs. Plates were sometimes incubated anaerobically with the AnaeroPack ${ }^{\circledR}$ system (Mitsubishi Gas Chemical Co., Tokyo, Japan) to recover strictly and facultatively anaerobic bacteria according to the manufacturer's instructions. Preliminary experiments showed that PBYG agar medium recovered higher VBCs from SCM than any other agar media tested, including Brain Heart Infusion (Difco), Nutrient Broth (Difco), Trypticase Soy Broth (Difco), and PBY medium (Hiraishi and Komagata, 1989). For measuring VMCs, each dilution was inoculated onto agar plates containing Malt Extract Agar (Difco) supplemented with $0.1 \% \mathrm{NaCl}(\mathrm{pH} \mathrm{4.8)}$. Incubation was at 28 and $50^{\circ} \mathrm{C}$ for 7 days.

Quinone analysis. SCM samples were thawed and washed twice with $50 \mathrm{~mm}$ phosphate buffer ( $\mathrm{pH}$ 6.8) containing $1 \mathrm{~mm}$ ferricyanide. Washed SCM (about $1 \mathrm{~g}$ wet wt) was mixed with $30 \mathrm{ml}$ of acetone, sonicated on ice for $1 \mathrm{~min}$ ( $20 \mathrm{kHz}$; output power $100 \mathrm{~W}$ ), and centrifuged at $8,000 \times g$ for $10 \mathrm{~min}$ to separate the acetone extract. The residue was then extracted three times with a chloroform-methanol mixture $(2: 1$, vol/vol $)$ by shaking for every $30 \mathrm{~min}$ and by filtrating through Whatman No. 1 filter papers. All extracts were combined, evaporated under vacuum, and reextracted three times with $n$-hexane-water $(1: 1, \mathrm{vol} / \mathrm{vol})$. The quinone extract in the hexane layer was collected, evaporated, and subjected to column chromatography with Sep-Pak Vac $3 \mathrm{cc}$ silica gel cartridge (Waters, Milford, MA, USA) to separate the menaquinone and ubiquinone fractions as described previously (Hiraishi et al., 1996; Iwasaki and Hiraishi, 1998). Quinone components in each fraction were separated and identified by reverse-phase HPLC and photodiode array detection with external quinone standards. Detailed information on the analytical procedure has been reported previously (Hiraishi et al., 1996; Iwasaki and Hiraishi, 1998). Furthermore, HPLC-mass spectrometry with the Hitachi M-8000 LC/3DQ. MS system (Hitachi Co., Tokyo, Japan) was sometimes used to confirm the molecular mass of quinone components. Standard quinones were prepared from known species of bacteria and activated sludge as described previously (Hiraishi et al., 1996). In this study, ubiquinones, menaquinones, and plastoquinones with $n$ isoprene units in their side chain were abbreviated as Q- $n$, MK$n$, and $P Q-n$, respectively. Partially hydrogenated ubiquinones and menaquinones were expressed as $Q$ $n\left(\mathrm{H}_{x}\right)$ and MK- $n\left(\mathrm{H}_{x}\right)$, respectively, where $x$ indicated the number of hydrogen atoms saturating the side chain. Phylloquinone (vitamin $\mathrm{K}_{1}$ ) was abbreviated as $K_{1}$. Furthermore, $U Q, M K$, and $P Q$ were used to call a total fraction of ubiquinones, menaquinones, and plastoquinones, respectively, as needed.

Numerical analysis. All quinone profiles obtained were recorded as mol\% and subjected to numerical analysis. The quinone profiles obtained previously for activated sludge (Hiraishi et al., 1996) and soil (Iwasaki and Hiraishi, 1998) were used as the reference data. Two parameters, the dissimilarity index $(D)$ (Hiraishi et al., 1991) and the microbial divergence index $\left(M D_{\mathrm{q}}\right)$ based on quinone profiles (Iwasaki and Hiraishi, 1998), were used for the numerical analysis. The dissimilarity index is defined as follows:

$$
D(i, j)=1 / 2 \sum_{k=1}^{n}\left|x_{k i}-x_{k j}\right|
$$

where $x_{k i}$ and $x_{k j}$ indicate mol\% of quinone homologue $k$ in samples $i$ and $j$, respectively. The microbial divergence index is given by

$$
M D_{\mathrm{q}}=\left(\sum_{k=1}^{n} \sqrt{x_{k}}\right)^{2}
$$

where $x_{k}$ indicates the molar ratio of quinone homologue $k$ to the total content as $1\left(x_{k} \geq 0.001\right)$. The $D$ and $M D_{\mathrm{q}}$ values were calculated, and a dendrogram based on $D$ matrix data was constructed by the neighbor-joining method (Saitou and Nei, 1987) with the BioCLUST program (Iwasaki and Hiraishi, 1998). The dendrogram was illustrated with the TreeView program (Page, 1996).

\section{Results}

\section{Performance and physico-chemical data}

The FUSBIC systems with 14-L and 20-L reactors were operated for the disposal of a total of $239 \mathrm{~kg}$ (wet wt) each of the biowaste collected over one year. The monthly averages or cumulative physico-chemical data obtained for the 14-L system during this period of operation are shown in Fig. 2. The primary reactors showed a monthly waste reduction rate of 75.3 to 
$97.1 \%$ with an average of $88.2 \%$. The reduction rate was lowered to less than $80 \%$ in January and June 1999. These observations could be explained by the rate of waste reduction in the FUSBIC system being affected by seasonal air temperature and weather

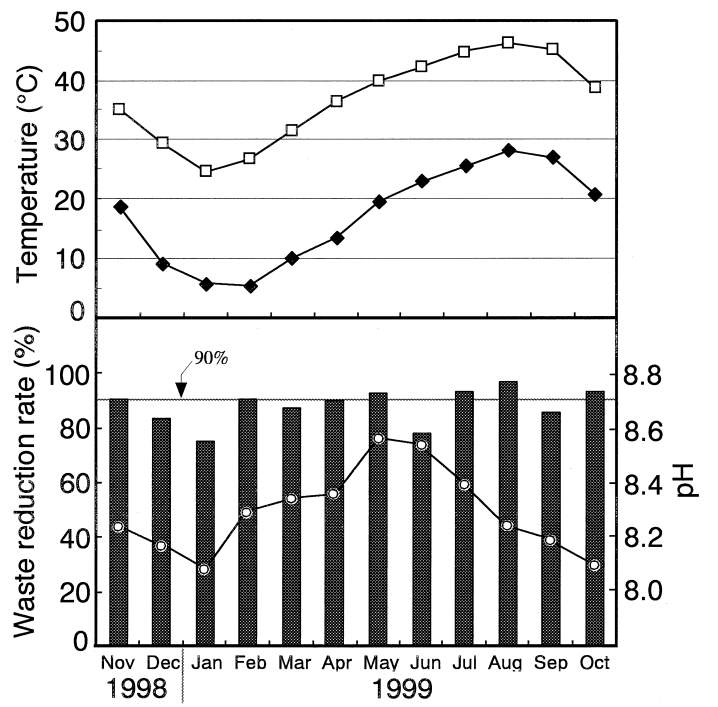

Fig. 2. Seasonal changes in waste reduction rate and some other physico-chemical characteristics of the FUSBIC system with 14-L reactors.

The waste reduction rate (\%) is shown by histograms. Symbols: $\diamond$, air temperature; $\square$, reactor temperature at $8 \mathrm{~cm}$ depth; $\mathrm{O}, \mathrm{pH}$.
(Hiraishi, 2000). That is, the decrease in the waste reduction rate in January and June 1999 was possibly due to the negative effects of low air temperature and the rainy season in Japan, respectively. The average waste reduction rate noted above corresponded to the production of about $28 \mathrm{~kg}$ wet wt of surplus compost by each system during the year, which was furthermore reduced by $19 \%$ through the secondary treatment (data not shown).

The monthly average $\mathrm{pH}$ of the SCMs was nearly constant regardless of season $(\mathrm{pH} 8.07-8.55)$; thereby the steady state of the reactors was found to be in slightly or moderately alkaline conditions. The monthly average temperature in the reactors ranged from 24.6 to $45.5^{\circ} \mathrm{C}$, being always 10 to $20^{\circ} \mathrm{C}$ higher than seasonal air temperature.

The FUSBIC system with 20-L reactors showed a monthly waste reduction rate of $92.5 \%$ during the period of operation (data not shown). The core temperature and $\mathrm{pH}$ in the 20 - $\mathrm{L}$ system were essentially the same as those found in the 14-L system.

\section{Microbial counts}

Microbial counts in the 14-L system were measured monthly on fine days through the year (Table 2). All SCM samples tested gave total counts of $10^{11}$ cells . $\mathrm{g}^{-1}$ of dry wt with a geometric mean of $6.7 \times 10^{11}$ cells .

Table 2. Microbial counts in the primary reactors of the FUSBIC system. ${ }^{a}$

\begin{tabular}{|c|c|c|c|c|c|c|c|}
\hline \multirow{2}{*}{ Date sampled } & \multirow{2}{*}{$\begin{array}{c}\mathrm{TC} \\
\left(\times 10^{11} \cdot \mathrm{g}^{-1}\right)\end{array}$} & \multicolumn{3}{|c|}{ VBC $\left(\mathrm{CFU} \times 10^{11} \cdot \mathrm{g}^{-1}\right)$ at: } & \multicolumn{2}{|c|}{ VMC $\left(\mathrm{CFU} \times 10^{9} \cdot \mathrm{g}^{-1}\right)$ at: } & \multirow{2}{*}{ VBC/TC ratio } \\
\hline & & $28^{\circ} \mathrm{C}$ & $37^{\circ} \mathrm{C}$ & $50^{\circ} \mathrm{C}$ & $28^{\circ} \mathrm{C}$ & $50^{\circ} \mathrm{C}$ & \\
\hline Nov. 23, 1998 & 7.6 & 4.5 & 4.2 & 0.027 & 1.1 & 0.010 & 0.59 \\
\hline Dec. 14,1998 & 6.3 & 4.1 & 2.9 & $n t^{b}$ & $\mathrm{nt}$ & nt & 0.65 \\
\hline Jan. 15, 1999 & 5.2 & 3.2 & 3.0 & 0.010 & 2.5 & 0.0020 & 0.62 \\
\hline Feb. 11, 1999 & 4.5 & 1.9 & 1.5 & 0.014 & $\mathrm{nt}$ & nt & 0.42 \\
\hline Mar. 23, 1999 & 7.1 & 2.8 & 1.1 & 0.009 & 2.8 & 0.0025 & 0.39 \\
\hline Apr. 16, 1999 & 6.6 & 4.9 & 2.9 & nt & nt & nt & 0.74 \\
\hline May 8, 1999 & 8.6 & 3.6 & 2.4 & 0.071 & 0.78 & 0.0012 & 0.42 \\
\hline June 15, 1999 & 8.9 & 3.8 & 2.1 & 0.036 & $\mathrm{nt}$ & $\mathrm{nt}$ & 0.43 \\
\hline July 23, 1999 & 9.6 & 4.8 & 4.0 & 0.080 & $\mathrm{nt}$ & $\mathrm{nt}$ & 0.54 \\
\hline Aug. 10, 1999 & 6.2 & 5.7 & 5.8 & 0.087 & 2.3 & 0.0054 & 0.92 \\
\hline Sept. 15, 1999 & 5.6 & 3.0 & 3.0 & 0.027 & nt & nt & 0.54 \\
\hline Oct. 11, 1999 & 6.0 & 2.7 & 2.6 & 0.041 & 3.5 & 0.011 & 0.45 \\
\hline Geometric mean & 6.7 & 3.6 & 2.6 & 0.030 & 1.9 & 0.0039 & $0.56^{c}$ \\
\hline
\end{tabular}

${ }^{a}$ Data show the count per g of dry wt of SCM.

${ }^{b} \mathrm{nt}$, not tested.

${ }^{c}$ Arithmetic mean. 


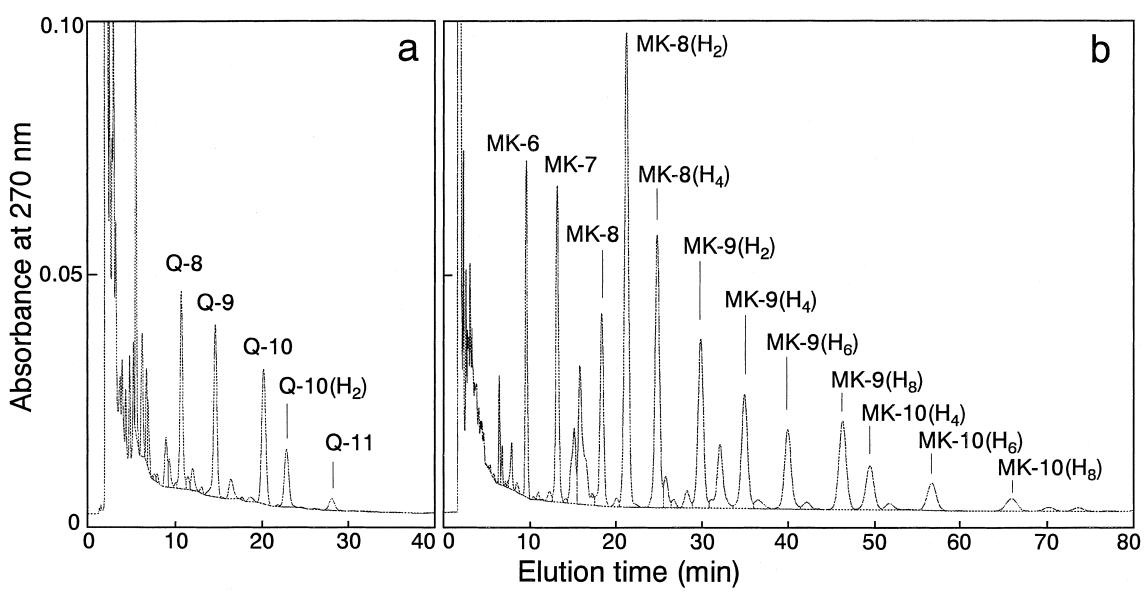

Fig. 3. Typical HPLC elution profiles of the quinones from an SCM sample.

(a) Ubiquinone fraction; (b) menaquinone fraction. Both fractions isolated from $1 \mathrm{~g}$ wet wt of SCM were dissolved in $100 \mu \mathrm{l}$ of acetone, and $10 \mu \mathrm{l}$ of each solution was injected to the HPLC apparatus. Quinone components were separated with Zorbax ODS column $(4.6 \times 250 \mathrm{~mm})$ at $30^{\circ} \mathrm{C}$ and eluted with methanol-isopropyl ether $(9: 2$, vol $/ \mathrm{vol})$ as the mobile phase at a flow rate of $1 \mathrm{ml} \cdot \mathrm{min}^{-1}$.

$\mathrm{g}^{-1}$ VBCs detected on PBYG agar media at $28^{\circ} \mathrm{C}$ were at the order of $10^{11}$ cells $\cdot \mathrm{g}^{-1}$ with a geometric mean of $3.6 \times 10^{11}$ cells $\cdot \mathrm{g}^{-1}$, accounting for about half of the TC. Similar or slightly lower levels of VBCs were recorded at $37^{\circ} \mathrm{C}$. VBCs at $50^{\circ} \mathrm{C}$ were much lower than those at $28^{\circ} \mathrm{C}$, but they could be detected constantly regardless of season. Attempts to detect anaerobic bacteria with the AnaeroPack system resulted in the recovery of lower counts than the VBCs of aerobic bacteria (data not shown). VMCs recorded at $28^{\circ} \mathrm{C}$ were 1 to $3 \%$ of the VBCs at $28^{\circ} \mathrm{C}$. It is interesting that all SCM samples tested produced low but detectable counts of molds at $50^{\circ} \mathrm{C}$.

The results of microbiological examinations indicated that thermophilic or thermotolerant species of bacteria and fungi alike were common members of the microbial population in the primary reactors, though the overwhelming majority of the inhabitants were mesophilic.

\section{Quinone analysis}

Before detailed analyses of quinone composition of SCMs, we examined the accuracy and reliability of the method of quinone analysis used. Examinations for quinone extraction from some SCMs with internal standards indicated that the efficiency for recovery of quinones was found to be more than $95 \%$. Furthermore, HPLC experiments of quinone mixtures extracted in 10 different runs from a single sample showed that the deviation in quinone composition of the same sample was less than $4.7 \%$, as expressed by $D$ values (mean \pm standard deviation $=2.37 \pm 1.28$ ). These results demonstrate not only that the accuracy and reproducibility of the method used is high, but also that more than $5 \%$ of the $D$ value is significant to indicate differences in quinone profiles.

Examples of HPLC elution patterns of a quinone mixture from an SCM sample are shown in Fig. 3. In general, the ubiquinone fraction generated three major peaks that corresponded to Q-8, Q-9, and Q-10 (Fig. 3a). Moreover, this fraction was characterized by constant production of a partially hydrogenated component eluted behind $\mathrm{Q}-10$. This component was identified as Q-10 $\left(\mathrm{H}_{2}\right)$ on the basis of its elution time, UV absorption spectrum, and mass spectrum $(\mathrm{M}+\mathrm{H}$, $m / z=865$ ). The elution pattern of the menaquinone fraction was much more complicated than that of the ubiquinone fraction (Fig. 3b). Especially, a number of elution peaks corresponding to partially hydrogenated components were usually detected with $\mathrm{MK}-8\left(\mathrm{H}_{2}\right)$ as the highest peak.

\section{Changes in quinone contents}

Seasonal changes in quinone contents of the SCM samples from the 14-L and 20-L systems are shown in Table 3. The quinone concentration in the SCMs ranged from 160 to $353 \mathrm{nmol} \cdot \mathrm{g}^{-1}$ of dry wt with an average of $223 \mathrm{nmol} \cdot \mathrm{g}^{-1}$ for the $14-\mathrm{L}$ system. This average quinone concentration corresponds to a total bacterial count of $6.7 \times 10^{11}$ cells $\cdot \mathrm{g}^{-1}$ if the statistical correlation between the two (Hiraishi, 1999b) is considered. And this estimated TC level based on the quinone con- 
Table 3. Quinone contents of SCMs (dry weight basis) from the primary reactors.

\begin{tabular}{|c|c|c|c|c|c|c|c|c|c|c|}
\hline \multirow{3}{*}{ Date sampled } & \multicolumn{5}{|c|}{ 14-L system } & \multicolumn{5}{|c|}{ 20-L system } \\
\hline & \multirow{2}{*}{$\begin{array}{c}\text { Total } \\
\text { content } \\
\left(\mathrm{nmol} \cdot \mathrm{g}^{-1}\right)\end{array}$} & \multicolumn{3}{|c|}{ Mol\% of: ${ }^{a}$} & \multirow{2}{*}{$\begin{array}{c}\text { MK/UQ } \\
\text { ratio }\end{array}$} & \multirow{2}{*}{$\begin{array}{c}\text { Total } \\
\text { content } \\
\left(\mathrm{nmol} \cdot \mathrm{g}^{-1}\right)\end{array}$} & \multicolumn{3}{|c|}{ Mol\% of: } & \multirow{2}{*}{$\begin{array}{c}\text { MK/UQ } \\
\text { ratio }\end{array}$} \\
\hline & & UQ & MK- $n$ & $\mathrm{MK}-n\left(\mathrm{H}_{x}\right)$ & & & UQ & MK- $n$ & MK- $n\left(\mathrm{H}_{x}\right)$ & \\
\hline Nov. 23, 1998 & 205 & 34.5 & 19.2 & 43.2 & 1.81 & $-b$ & - & - & - & - \\
\hline Dec. 14, 1998 & 198 & 34.0 & 18.4 & 44.0 & 1.84 & 211 & 33.0 & 17.7 & 46.2 & 1.94 \\
\hline Jan. 15, 1999 & 160 & 36.4 & 18.5 & 41.8 & 1.65 & - & - & - & - & - \\
\hline Feb. 11, 1999 & 182 & 35.8 & 16.9 & 45.7 & 1.73 & 208 & 32.9 & 19.1 & 44.6 & 1.93 \\
\hline Mar. 23, 1999 & 210 & 33.9 & 16.3 & 45.7 & 1.83 & - & - & - & - & - \\
\hline Apr. 16, 1999 & 208 & 27.4 & 18.8 & 51.3 & 2.56 & 215 & 30.5 & 18.0 & 49.7 & 2.22 \\
\hline May 8, 1999 & 256 & 15.0 & 21.3 & 61.6 & 5.51 & - & - & - & - & - \\
\hline June 15, 1999 & 305 & 16.9 & 22.0 & 60.1 & 4.87 & 253 & 25.3 & 18.2 & 54.5 & 2.87 \\
\hline July 23, 1999 & 353 & 23.8 & 20.7 & 54.4 & 3.16 & - & - & - & - & - \\
\hline Aug. 10, 1999 & 242 & 27.2 & 14.8 & 57.4 & 2.65 & 196 & 28.2 & 17.0 & 54.0 & 2.52 \\
\hline Sept. 15, 1999 & 173 & 29.3 & 15.9 & 54.1 & 2.39 & - & - & - & - & - \\
\hline Oct. 11, 1999 & 189 & 26.3 & 15.0 & 57.8 & 2.77 & 210 & 28.3 & 15.7 & 55.2 & 2.51 \\
\hline Mean \pm SD & $223 \pm 57.0$ & $28.4 \pm 7.1$ & $18.2 \pm 2.4$ & $51.4 \pm 7.1$ & $2.73 \pm 1.25$ & $216 \pm 19.5$ & $29.7 \pm 3.0$ & $17.6 \pm 1.2$ & $50.7 \pm 4.6$ & $2.33 \pm 0.37$ \\
\hline
\end{tabular}

${ }^{a} \mathrm{UQ}, \mathrm{MK}-n$, and MK- $n\left(\mathrm{H}_{x}\right)$ indicate the fraction of total ubiquinones, unsaturated menaquinones, and partially hydrogenated menaquinones, respectively.

$b_{-}$, not tested.

centration agrees well with the geometric mean of TC actually determined for the 14-L system (see Table 2).

All SCM samples tested were characterized by the production of much higher amounts of menaquinones, especially partially saturated menaquinones, than of ubiquinones. Some variations were noted in the proportion of ubiquinones, unsaturated menaquinones, and partially saturated menaquinones to the total quinone content, as well as in MK/UQ molar ratios. These variations were more pronounced in the 14-L reactors than in the $20-\mathrm{L}$ reactors. In particular, a marked increase in MK/UQ ratios were found in the 14-L system in May to June 1999. We have not yet found the definitive reason for the degree of variations in quinone profiles being larger in the $14-\mathrm{L}$ system. But it may be assumed that the microbial communities of smaller reactors are more affected by weather, air temperature, and other environmental factors.

More detailed information on the quinone profiles is shown in Table 4. As noted above, some differences were found between the 14-L and 20-L systems in the degree of variations in quinone profiles. But in both systems, similar major types of quinones were detected as the primary and the second most common components during the period of operation. These major types were MK- $8\left(\mathrm{H}_{2}\right)$, MK- $9\left(\mathrm{H}_{2}\right)$, and Q-10. Thus no essential difference was noted in the averaged quinone profiles between the two systems, as shown in Fig. 4. Based on the available chemotaxonomic data (Collins and Jones, 1981; Hiraishi, 1999b), it could be assumed that Q-10 and all other unsaturated ubiquinone types were originated from species of the Proteobacteria and fungi, partially saturated and long-chain menaquinones ( $\geq \mathrm{MK}-8$ ) from species of the Actinobacteria (gram-positive bacteria with a high $\mathrm{G}+\mathrm{C}$ content), and lower homologues of menaquinones ( $\leq \mathrm{MK}-8$ ) from gram-positive bacteria with a low $\mathrm{G}+\mathrm{C}$ content and other phylogenetic groups of bacteria. It was apparent that $\mathrm{Q}-10\left(\mathrm{H}_{2}\right)$ was derived from some species of yeasts and fungi. The low contents of $P Q$ and $K_{1}$ detected might be derived from the residual biowaste (e.g., vegetables and tea grounds) rather than from oxygenic photosynthetic microorganisms.

\section{Numerical analyses of quinone profiles}

To obtain quantitative information on seasonal changes in the whole community structure in the primary reactors, we made numerical analyses of quinone profiles by using the two parameters, $D$ and 


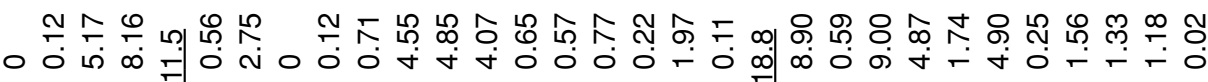

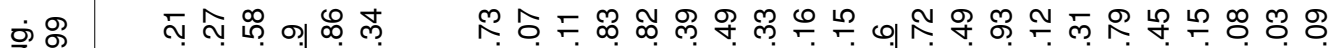
₹응 ○

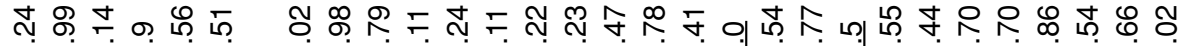
○

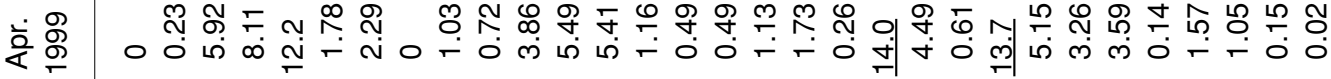

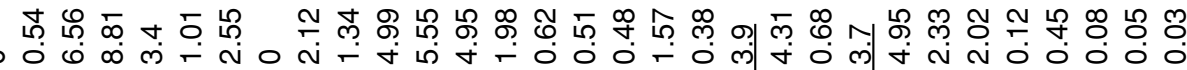
ن $\stackrel{\mathscr{\Phi}}{\circ} \stackrel{0}{\circ}$

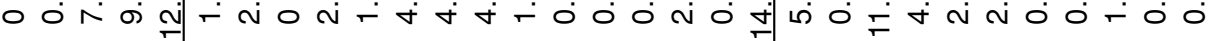

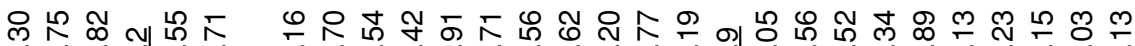

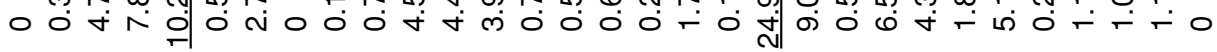

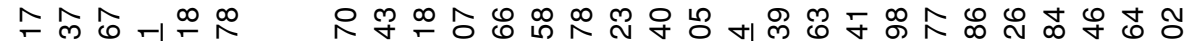
○ 0 ○

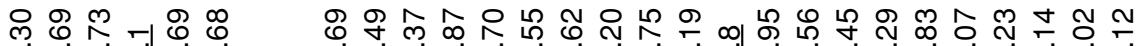
○ 0 ம

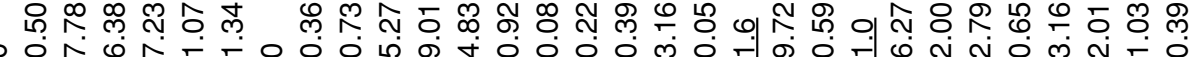

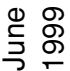

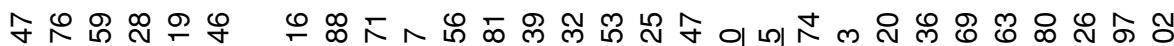
○

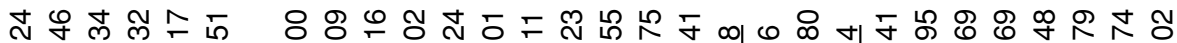
○ 0 N

임 ○ 0 ம

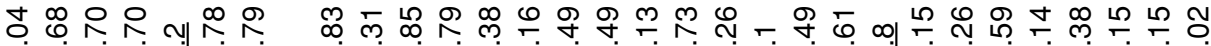
○ 0 0 ఫ

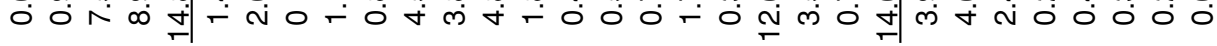

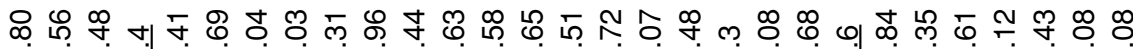

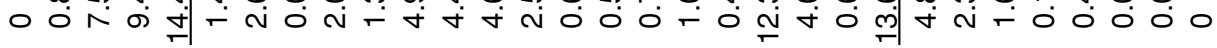

ఫ ○ O

ஜ 


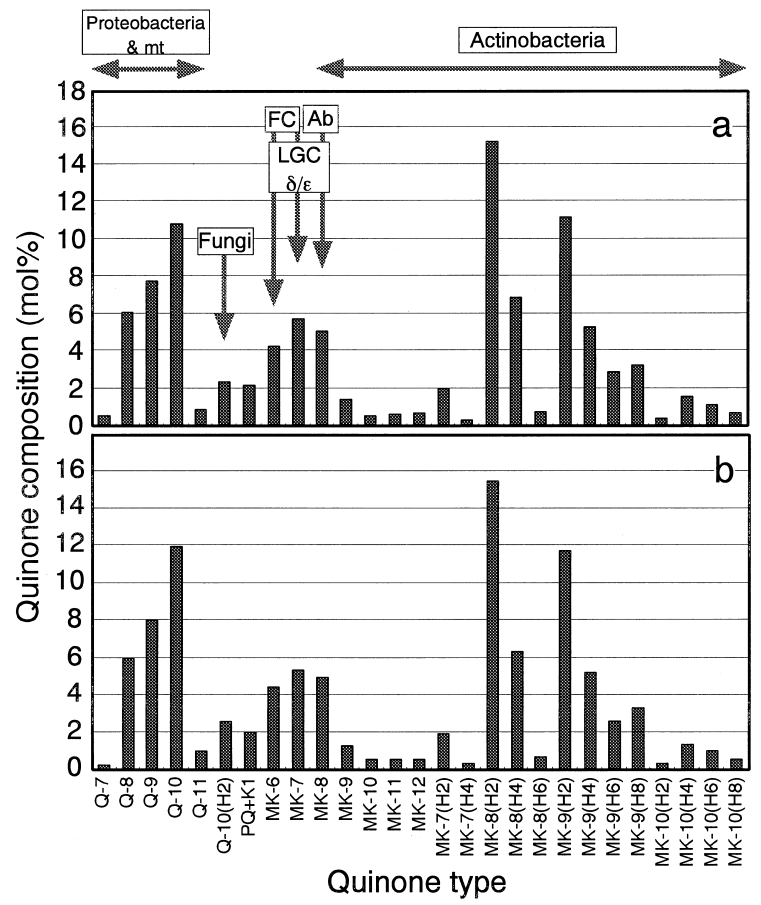

Fig. 4. Averaged quinone composition of the SCMs from the 14- $\mathrm{L}$ system (a) and 20-L system (b).

Phylogenetic groups of microbes as possible sources of each quinone type are shown. Abbreviations: FC, FlavobacteriumCytophaga group; LGC, gram-positive bacteria with a low $\mathrm{G}+\mathrm{C}$ content; $\delta / \varepsilon, \delta$ - and $\varepsilon$-Proteobacteria; Ab, Acidobacterium group; $\mathrm{mt}$, mitochondria.

$M D_{\mathrm{q}}$. Table 5 summarizes $D$ matrix data and $M D_{\mathrm{q}}$ values calculated for all SCM samples tested. Binary $D$ values for all the samples ranged from 1.1 to 30.9 with an average \pm standard deviation of $17.0 \pm 6.9$. Therefore the upper limit of seasonal variations in microbial communities as shown by $D$ values was evaluated to be about $30 \%$. The $M D_{\mathrm{q}}$ values calculated were 18.9 to 23.6 (average, 20.4). These $M D_{\mathrm{q}}$ values are much higher than those recorded for other environments (Hiraishi, 1999b; Iwasaki and Hiraishi, 1998).

Based on the $D$ matrix data obtained with all SCM samples and activated sludge and soil samples used as the reference, a neighbor-joining dendrogram was constructed (Fig. 5). The microbial communities from the 14-L and 20-L systems formed a single cluster clearly separable from the cluster of the sewage-activated sludge and the soil community used as the reference. As judged from the length of trees in each cluster, the seasonal divergence of communities was larger in the composting system than in the activated sludge system. In the 14-L system, the SCM communities different over time were classified into three major subclusters, i.e., those from November 1998 to April 1999 (subcluster I), those from May to July 1999 (subcluster II), and those from August to October 1999 (subcluster III). The dendrogram indicates that the most pronounced population shift occurred from April (subcluster I) to May 1999 (subcluster II), in which the highest MK/UQ ratio was found (see Table 3).

Correlation between temperature and microbiological parameters

The correlation coefficients between air and SCM temperature and different microbiological parameters were calculated to obtain more definite information on seasonal microbial community changes in the FUSBIC system. As shown in Table 6, TC, VBC, total quinone and MK contents, and MK/UQ ratios correlated positively with the temperature, whereas there seemed to be a negative correlation between the UQ content and the temperature. Among these relationships, however, only the correlation between the partially saturated MK content and the temperature was significant at the $95 \%$ level.

\section{Discussion}

The results of microbiological examinations in this study have shown that high densities of microorganisms $\left(10^{11}\right.$ cells $\cdot \mathrm{g}^{-1}$ of dry SCM) are present in the primary reactors of the FUSBIC system, regardless of season. The geometric mean of the TCs detected by epifluorescence microscopy was $6.7 \times 10^{11}$ cells $\cdot \mathrm{g}^{-1}$. Moreover, the aerobic enumeration with PBYG agar plates at $28^{\circ} \mathrm{C}$ gave the highest levels of VBCs with a geometric mean of $3.6 \times 10^{11} \mathrm{CFU} \cdot \mathrm{g}^{-1}$ among the experimental conditions tested. The VBC thus obtained accounted for about half of the TC. These data indicate that such aerobic, mesophilic, and chemoorganotrophic bacteria as recoverable under the cultivation conditions we used are the major constituents of the microbial population in the FUSBIC system. Because no detailed information on the direct total count and culturability for the predominant bacteria in compost has been found before this study, it is difficult to compare our data with the data of previous reports. The VBCs obtained in this study are higher than those recorded previously on some composting systems (Beffa et al., 1996; Nakasaki et al., 1998; Strom, 1985a).

In natural aquatic and terrestrial environments, 


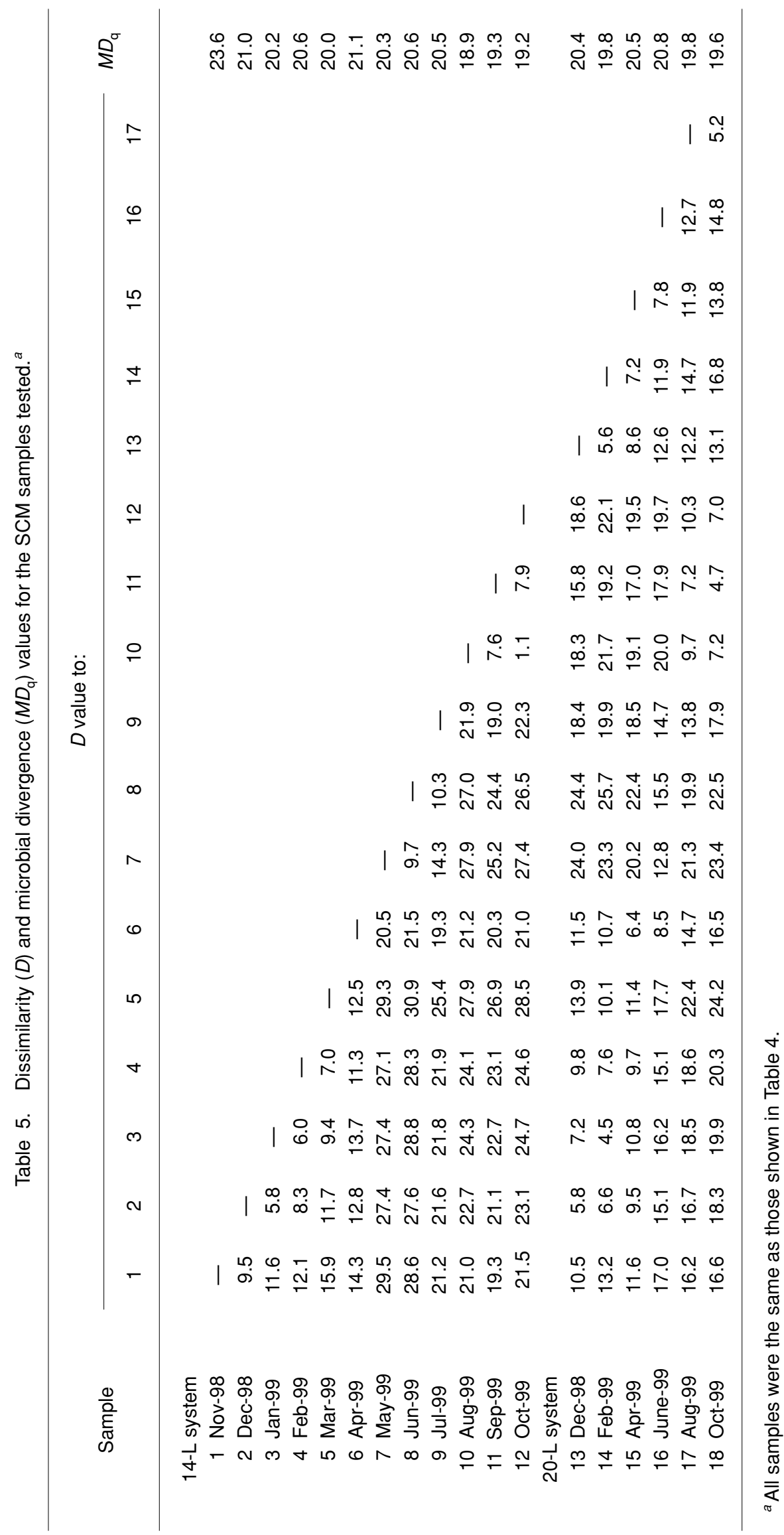




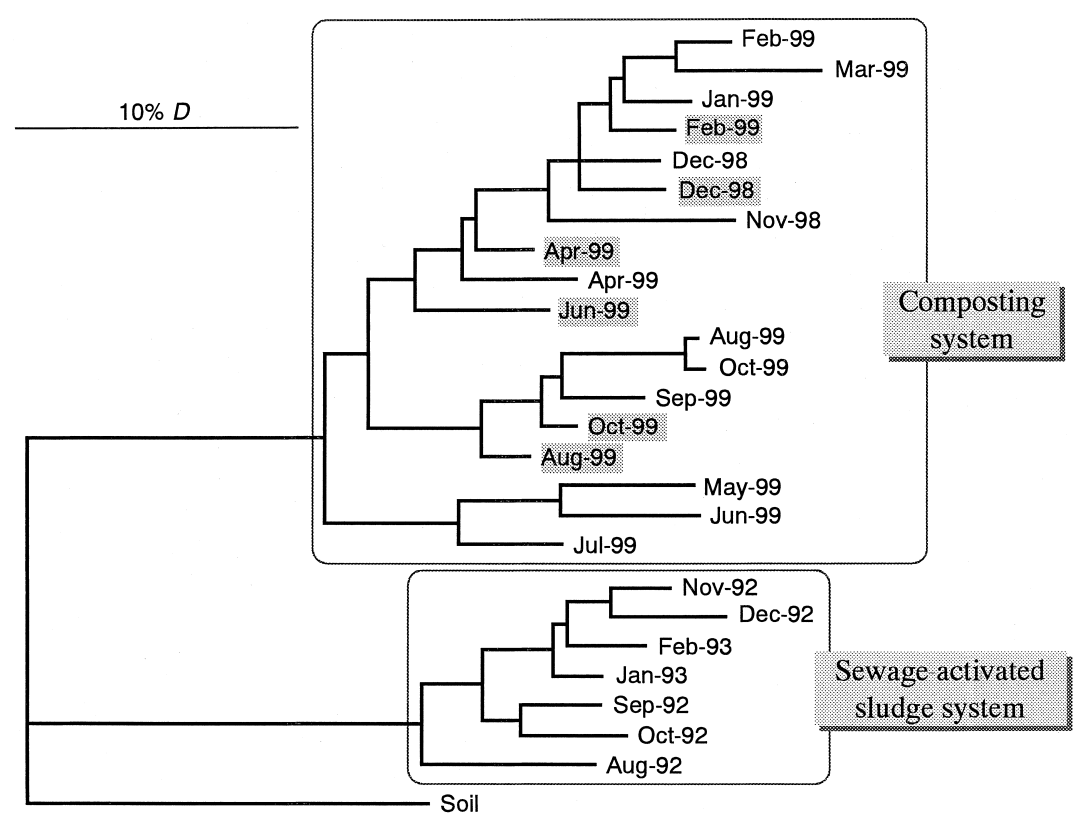

Fig. 5. Neighbor-joining dendrogram showing relationships among quinone profiles of SCM samples studied.

The samples from the 20-L system are shaded. The quinone profiles of sewage-activated sludge (Hiraishi et al., 1996) and soil (Iwasaki and Hiraishi, 1998) were used as the reference data. Scale $=10 \%$ divergence as $D$ value.

Table 6. Correlation coefficients between seasonal temperature and microbiological parameters. ${ }^{a}$

\begin{tabular}{lcc}
\hline & \multicolumn{2}{c}{ Correlation coefficient $(r)$ to: } \\
\cline { 2 - 3 } Parameter $^{b}$ & Air temperature & SCM temperature \\
\hline TC & 0.4840 & 0.4886 \\
VBC & 0.4471 & 0.4499 \\
TQ & 0.5394 & 0.5573 \\
UQ & -0.1029 & -0.1818 \\
MK- $n\left(\mathrm{H}_{0}\right)$ & 0.4060 & 0.4313 \\
MK- $n\left(\mathrm{H}_{x}\right)$ & 0.6605 & 0.6995 \\
MK/UQ & 0.4911 & 0.5661 \\
\hline
\end{tabular}

${ }^{a} n=12$. Values significant at $\geq 95 \%$ level are asterisked.

${ }^{b}$ Abbreviations for parameters: TC, total count; VBC, viable bacterial count; $\mathrm{TQ}$, total quinone content; $\mathrm{UQ}$, ubiquinone content; MK- $n\left(\mathrm{H}_{0}\right)$, unsaturated menaquinone content; MK- $n\left(\mathrm{H}_{x}\right)$, partially saturated menaquinone content $(x \geq 2)$; MK/UQ, molar ratio of menaquinones to ubiquinones.

$\mathrm{VBC} / \mathrm{TC}$ ratios are very low, usually less than $1 \%$. Even in activated sludge systems, VBCs account only for 1 to $15 \%$ of the total population (Amann et al., 1995). Because of these low VBC/TC ratios and possible culture biases, culture-independent molecular techniques have been used for the characterization of bacterial communities in these environments. Compared with natural and wastewater environments, the FUSBIC system gave much higher $\mathrm{VBC} / \mathrm{TC}$ ratios, as reported here and previously (Hiraishi, 1999a, 2000). The composting reactor can be regarded as a cultivation system with high concentrations of organic compounds, which is similar in nutrient composition and loading level to microbial culture media routinely used in laboratories. This is possibly why most bacteria from the composting process are culturable, unlike bacterial populations in natural environments. So although it is difficult to describe the microbial community fully by cultivation methods, a culture-dependent approach may be much more useful for the study of composting systems than for the study of natural and other engineered environments. In this connection, chemotaxonomic and phylogenetic analyses of the microbial strains isolated from the FUSBIC system are now in progress.

The analysis of respiratory quinones from the FUSBIC system in this study confirmed the previous results (Hiraishi, 1999a, 2000) and expanded the information on the microbial community structure in the composting system. One of the characteristic features of the FUSBIC system is the production of much higher amounts of menaquinones than of ubiquinones. The average proportions of ubiquinones, unsaturated menaquinones, and partially saturated menaquinones 
to the total content were $28.4,18.1$, and $51.4 \mathrm{~mol} \%$, respectively (Table 3 ). The available chemotaxonomic information has shown that long-chained menaquinones and partially hydrogenated menaquinones are distributed mainly in species of the Actinobacteria (Collins and Jones, 1981; Hiraishi, 1999b). Therefore it may be assumed that members of the Actinobacteria account for more than $50 \%$ of the microbial population in the steady state of the primary reactors in the FUSBIC process. The presence and some ecological roles of the Actinobacteria in composting processes have been shown by several investigators (Blanc et al., 1997; Finstein and Morris, 1975; Fogarty and Tuovinen, 1991; Peters et al., 2000). The quinone profile data show that species with MK-8 $\left(\mathrm{H}_{2}\right)$, MK$9\left(\mathrm{H}_{2}\right)$, and some other MK- $n\left(\mathrm{H}_{x}\right)$ types are the major constituents of the actinobacterial population present.

Lower homologues of unsaturated menaquinones (MK-6 to MK-8) found in the FUSBIC system may be derived from gram-positive bacteria with a low $\mathrm{G}+\mathrm{C}$ content, such as Bacillus species, and some other phylogenetic groups of bacteria. It has been shown that thermophilic Bacillus species, which contain MK-7 as the major quinone, are typical members of the microbial population in hot compost and in a composter operated at high temperature (Blanc et al., 1997; Pedro et al., 1999; Peters et al., 2000; Strom, 1985b). Thermogenic compost is also a rich source of Thermus species (Beffa et al., 1996), which contain MK-8 as the major component. Other candidates as possible producers of lower homologues of menaquinones are members of $\delta$ - and $\varepsilon$-Proteobacteria, the Flavobacterium-Cytophaga group, and the Acidobacterium group.

The occurrence of $Q-8, Q-9$, and $Q-10$ in large amounts in the FUSBIC system suggests that $\alpha-, \beta-$, and $\gamma$-Proteobacteria constitute a significant proportion of the microbial population present in the steady state. Some portions of the amounts of Q-9 and Q-10 might be derived from yeasts and fungi. Furthermore, the detection of partially hydrogenated ubiquinones apparently indicates the presence of certain species of yeasts and fungi, though the plate-counting data suggested that yeasts and fungi occurred in small populations in the FUSBIC system as the VMCs recorded were only 1 to $3 \%$ of the VBCs. The cultivation method used, however, might underestimate the population of yeasts and fungi because of the amounts of partially hydrogenated ubiquinones and other ubiquinone types detected.

The numerical analysis of quinone profile data indicates that the maximum level of seasonal variations in the microbial community structure in the FUSBIC process was about $30 \%$ as the $D$ value. This range of the variation is somewhat larger than that for sewageactivated sludge, as reported here. Since the FUSBIC system is an open composting process not controlled by any electric apparatus, its performance is strongly affected by weather, air temperature, and other environmental factors (Hiraishi, 2000). This is one of the most plausible reasons why the microbial community structure in FUSBIC system changes more than that in activated sludge systems. The statistical analysis of the seasonal data indicated that microbial populations containing partially saturated menaquinones, i.e., actinobacterial populations, vary significantly in response to seasonal temperature. The finding that the microbial communities in the winter and summer seasons formed different clusters on the neighbor-joining dendrogram can be explained primarily by the variations in actinobacterial populations.

The $M D_{\mathrm{q}}$ levels recorded for the FUSBIC system are much higher than those for activated sludge systems and natural environments so far studied for quinone profiles (Hiraishi and Kato, 1999; Hiraishi et al., 1998, 1999; Iwasaki and Hiraishi, 1998). These numerical data, together with the results of the community analysis, allow us to draw the obvious conclusion that the resident microbial community consists of highly diverse taxonomic groups with actinobacterial populations as the major constituents.

As reported here, changes in the whole community structure in the composting system can be evaluated quantitatively by quinone profiling and the subsequent numerical analysis. Especially, the cluster analysis with neighbor-joining dendrograms is quite useful to determine the structural relationships among different microbial communities over time and space. Since the quinone profile method is a direct chemical analysis of lipid molecules, this method gives more reproducible and less-biased results than molecular techniques involved with nucleic acid extraction and PCR, which are now widely used for analyzing complex microbial communities. A combined use of the quinone profile method and molecular and ecophysiological techniques should therefore provide more useful information on the microbiology of compost. 


\section{Acknowledgments}

We are grateful to the staff of the restaurant of Toyohashi University of Technology for providing organic waste. Special thanks go to the author's (A.H.) family for the maintenance of the reactors.

\section{References}

Amann, R. L., Ludwig, W., and Schleifer, K. H. (1995) Phylogeneticidentification and in situ detection of individual microbial cells without cultivation. Microbiol. Rev., 59, 143-169.

Beffa, T., Blanc, M., Lyon, P. F., Vogt, G., Marchiani, M., Fischer, J. L., and Aragno, M. (1996) Isolation of Thermus strains from hot composts $\left(60\right.$ to $\left.80^{\circ} \mathrm{C}\right)$. Appl. Environ. Microbiol., 62, 1723-1727.

Blanc, B., Marilley, L., Beffa, T., and Aragno, M. (1997) Rapid identification of heterotrophic, thermophilic, spore-forming bacteria isolated from hot composts. Int. J. Syst. Bacteriol., 47, 1246-1248.

Carpenter-Boggs, L., Kennedy, A. C., and Reganold, J. P. (1998) Use of phospholipid fatty acids and carbon source utilization patterns to track microbial community succession in developing compost. Appl. Environ. Microbiol., 64, 4062-4064.

Collins, M. D. and Jones, D. (1981) Distribution of isoprenoid quinone structural types in bacteria and their taxonomic implications. Microbiol. Rev., 45, 316-354.

Finstein, M. S. and Morris, M. L. (1975) Microbiology of municipal solid waste composting. Adv. Appl. Microbiol., 18, 113-151.

Fogarty, A. M. and Tuovinen, O. H. (1991) Microbiological degradation of pesticides in yard waste composting. Microbiol. Rev., 55, 225-233.

Fujie, K., Hu, H.-Y., Tanaka, H., Urano, K., Saito, K., and Katayama, A. (1998) Analysis of respiratory quinones in soil for characterization of microbiota. Soil Sci. Plant Nutr., 44, 393-404.

Hellmann, B., Zelles, L., Palojärvi, A., and Bai, Q. (1997) Emission of climate-relevant trace gases and succession of microbial communities during open-window composting. Appl. Environ. Microbiol., 63, 1011-1018.

Herrmann, R. F. and Shann, J. F. (1997) Microbial community changes during the composting of municipal solid waste. Microb. Ecol., 33, 78-85.

Hiraishi, A. (1999a) A simple composting system with flowerpots and garden soil for disposal of household organic waste. Seibutsu-kogaku Kaishi, 77, 493-500 (in Japanese).

Hiraishi, A. (1999b) Isoprenoid quinones as biomarkers of microbial populations in the environment. J. Biosci. Bioeng., 88, 415-436.

Hiraishi, A. (2000) Effects of weather and other environmental factors on the performance of a composting system using flowerpots for disposal of household biowaste. Seibutsu- kogaku Kaishi, 78, 301-310 (in Japanese).

Hiraishi, A. and Kato, K. (1999) Quinone profiles in lake sediments: Implications for microbial diversity and community structures. J. Gen. Appl. Microbiol., 45, 221-227.

Hiraishi, A. and Komagata, K. (1989) Effects of the growth medium composition on menaquinone homolog formation in Micrococcus luteus. J. Gen. Appl. Microbiol., 35, 311318.

Hiraishi, A., Morishima, Y., and Takeuchi, J. (1991) Numerical analysis of lipoquinone patterns in monitoring bacterial community dynamics in wastewater treatment systems. J. Gen. Appl. Microbiol., 37, 57-70.

Hiraishi, A., Ueda, Y., and Ishihara, J. (1998) Quinone profiling of bacterial populations in natural and synthetic sewage activated sludge for enhanced phosphate removal. Appl. Environ. Microbiol., 64, 992-998.

Hiraishi, A., Ueda, Y., Ishihara, J., and Mori, T. (1996) Comparative lipoquinone analysis of influent sewage and activated sludge by high-performance liquid chromatography and photodiode array detection. J. Gen. Appl. Microbiol., 42, 457-469.

Hiraishi, A., Umezawa, T., Yamamoto, H., Kato, K., and Maki, Y. (1999) Changes in quinone profiles of hot spring sulfur-turf microbial mats with a thermal gradient. Appl. Environ. Microbiol., 65, 198-205.

Insam, H., Amor, K., Renner, M., and Crepaz, C. (1996) Changes in functional abilities of the microbial community during composting of manure. Microb. Ecol., 31, 77-87.

Iwasaki, M. and Hiraishi, A. (1998) A new approach to numerical analyses of microbial quinone profiles in the environment. Microbes Environ., 13, 67-76.

Katayama, A., Hu, H.-Y., Nozawa, M., Yamakawa, H., and Fujie, K. (1998) Long-term changes in microbial community structure in soils subjected to different fertilizing practices revealed by quinone profile analysis. Soil Sci. Plant Nutr., 44, 559-570.

Klamer, M. and Bååth, E. (1998) Microbial community dynamics during composting of straw material studied using phospholipid fatty acid analysis. FEMS Microbiol. Ecol., 27, 9-20.

Kowalchuk, G. A., Naoumenko, Z. S., Derikx, P. J. L., Felske, A., Stephen, J. R., and Arkhipchenko, I. A. (1999) Molecular analysis of ammonia-oxidizing bacteria of the $\beta$ subdivision of the class Proteobacteria in compost and composted materials. Appl. Environ. Microbiol., 65, 396-403.

Nakasaki, K., Hiraoka, S., and Nagata, H. (1998) A new operation for producing disease-suppressive compost from grass clippings. Appl. Environ. Microbiol., 64, 4015-4020.

Page, R. D. M. (1996) TREEVIEW: An application to display phylogenetic trees on personal computers. Comput. Appl. Biosci., 12, 357-358.

Pedro, M. S., Hayashi, N. R., Mukai, T., Ishii, M., Yokota, A., and Igarashi, Y. (1999) Physiological and chemotaxonomical studies on microflora within a compostor operated at high temperature. J. Biosci. Bioeng., 88, 92-97. 
Peters, S., Koschinsky, S., Schwieger, F., and Tebbe, C. C. (2000) Succession of microbial communities during hot composting as detected by single-strand-conformation polymorphism-based genetic profiles small-subunit rRNA genes. Appl. Environ. Microbiol., 66, 930-936.

Roser, D. J. (1980) Ethidium bromide: A general purpose fluorescent stain for nucleic acid in bacteria and eucaryotes and its use in microbial ecology studies. Soil Biol. Biochem., 12, 329-336.

Saitou, N. and Nei, M. (1987) The neighbor-joining method: A new method for reconstructing phylogenetic trees. Mol.
Biol. Evol., 4, 406-425.

Someya, T. (1995) Three-dimensional observation of soil bacteria in organic debris with a confocal laser scanning microscope. Soil Microorg., 46, 61-69.

Strom, P. F. (1985a) Effect of temperature on bacterial species diversity in thermophilic solid-waste composting. Appl. Environ. Microbiol., 50, 899-905.

Strom, P. F. (1985b) Identification of thermophilic bacteria in solid-waste composting. Appl. Environ. Microbiol., 50, 906-913. 